\title{
The biosimilar name debate: what's at stake for public health
}

Emily A Alexander, JD

\section{As the number of innovator biologics and biosimilars increases worldwide, a growing debate has focused on how these products should be named. The simple con- cept of a name can have significant impact on prescrib- ing, dispensing, and adverse event reporting processes and, consequently, patient safety.}

Keywords: Adverse event reporting, biosimilars, naming

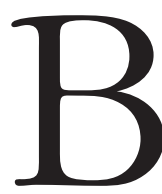

iosimilars have now been on the market in the European Union for more than six years, and several other jurisdictions have approved biosimilars as well. Biosimilars are poised to enter the US market as soon as 2014, and more countries are developing approval pathways for biosimilars each year. As part of the discussion of the regulatory and scientific issues surrounding biosimilars, attention has increasingly turned to how all biologicals should be named and, in particular, whether biosimilars should have non-proprietary names that distinguish them from the reference product and from other biosimilars.

Background on biosimilar naming conventions to date

Thus far, the approaches to biosimilar

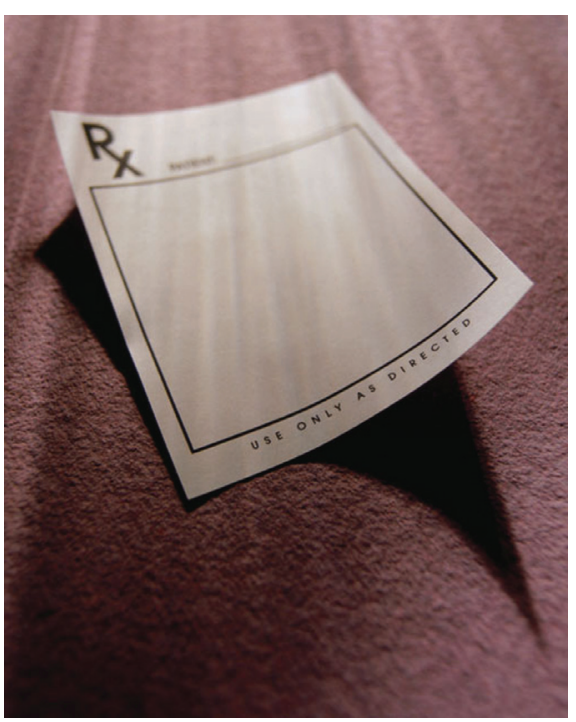

naming have been somewhat inconsistent and are becoming more complex. The World Health Organization (WHO) has historically assigned biosimilars International Nonproprietary Names (INNs) that are the same as those of the reference products. However, a biosimilar could elect to use a Greek letter suffix to indicate potential differences in glycoforms when compared to the reference product [1]. National health authorities generally do not require 
a biosimilar applicant to submit an application to the WHO International Nonproprietary Name Committee for evaluation.

Biosimilars in Europe have generally (but not always) shared the non-proprietary names of their reference products. For example, Silapo (epoetin zeta) has a distinct non-proprietary name from its reference product, Eprex/Erypo (epoetin alfa) [2]. Remsima (infliximab), in contrast, shares the same non-proprietary name as its reference product, Remicade (infliximab) [3].

As regulators, physicians, and patients have gained experience with biosimilars and manufacturers have begun developing more complex biosimilars like monoclonal antibodies, support has grown for giving them distinct non-proprietary names, i.e. names that distinguish biosimilars from their reference products and from each other. For example, Australia's Therapeutic Goods Administration (TGA) recently issued guidance requiring that the non-proprietary name of a biosimilar be composed of: (1) the reference product non-proprietary name; and (2) a biosimilar identifier, consisting of the prefix 'sim' and a three letter unique identifier code. According to TGA, this unique identifier code would be issued by the WHO International Nonproprietary Name Committee [4].

Japan also requires that biosimilars of complex protein products bear unique nonproprietary names. The naming convention there requires the biosimilar to use the nonproprietary name of the reference product, plus 'biosimilar' and a number indicating the order in which the biosimilar was approved in Japan, e.g. 1, 2, $3[5,6]$.

In the absence of a specific biosimilar naming scheme mandated by statute, US Food and Drug Administration (FDA) is in the process of developing its policy on biosimilar naming. Meanwhile, the agency recently approved full, non-abbreviated applications for three biologicals bearing non-proprietary names with distinctive prefixes: tbo-filgrastim, ziv-aflibercept, and ado-trastuzumab emtansine. FDA reasoned that distinct names would facilitate postmarket safety monitoring and help minimize the potential for medication errors (by reducing the risk of a patient receiving a product different than what the physician intended and by reducing confusion among healthcare providers who may consider same names to mean that products are clinically indistinguishable) [7].
The current lack of global uniformity of non-proprietary naming of biosimilars may weaken the INN system, and the inconsistency could lead to confusion among stakeholders, including prescribers, pharmacists, patients, and the broader scientific community. WHO is now in the process of considering a scheme in which a unique identifier would be added to the non-proprietary name of a biosimilar, in order to distinguish it from the reference product and from other biosimilars [1].

Distinct non-proprietary names for all biologicals, including biosimilars, are essential to promoting the public health by helping to facilitate: (1) transparency and clear identification of a product; and (2) efficient and accurate pharmacovigilance.

\section{Clear identification of biological products}

Depending on the pharmacy laws and practices of a jurisdiction, shared non-proprietary names may increase the potential for automatic substitution at the pharmacy or lead to unintended switching from one product to another where a physician intended to prescribe a specific product. A physician may choose to prescribe a specific product for any number of reasons, including because individual patients may have been stabilized on a particular product. But in jurisdictions where prescribing by brand name is prohibited or discouraged, if non-proprietary names are shared across biologicals, including biosimilars, patients may end up receiving products that are different from those intended by physicians. This lack of transparency can pose threats to patients and the physician-patient relationship. Further, some countries base their procurement tenders on non-proprietary name, a practice that may lead to patients being switched (sometimes repeatedly) among non-interchangeable products that share the same name.

In addition, three recent surveys of prescribers in the US and European Union suggest that some physicians believe a shared nonproprietary name implies two products are structurally identical [8-10]. Shared names may therefore muddle prescriber choice by giving the impression that a biosimilar is identical to its reference product and identical to other biosimilars that share the same reference product, neither of which is the case. A biosimilar, by definition, is similar but not identical to the relevant reference product. Moreover, biosimilars will not undergo comparability testing with regard to other biosimilars. Without ensuring differentiation in naming among biosimilars, physicians or other stakeholders may be led to believe that different biosimilar products are identical to one another based solely on their individual biosimilarity to the reference product. In this way, shared nonproprietary names may inhibit physician understanding of the nature of biosimilars and may thwart physician efforts to ensure a patient receives the intended product.

Efficient and accurate pharmacovigilance Effective post-market surveillance, and ultimately corrective actions to address safety concerns, hinges on the ability to efficiently and accurately identify potential safety signals and the product(s) responsible. The complexity of biological products and their sensitivity to even seemingly minor manufacturing changes and to environmental conditions means that, in some cases, it is not possible to fully predict the effect of a manufacturing change before it is implemented. It also means that clinically meaningful differences between biosimilars and their reference products sometimes may be detected only, or indeed may emerge, after biosimilar approval. It is therefore critical to be able to efficiently identify any adverse events associated with a specific biological after it reaches the market.

Spontaneous reports of adverse events often do not include meaningful productidentifying information beyond a product's non-proprietary name [7, 11], and electronic payer databases vary in the extent to which they collect product-specific data. Shared non-proprietary names across products thus often result in the pooling of adverse events associated with multiple products, potentially masking a change in adverse events with respect to one product but not others as well as impeding the ability to identify the product(s) responsible for a safety signal [12].

In contrast, the distinct name approach currently under consideration by WHO would appropriately indicate that two related products are, in fact, related. But it would also indicate that two biologicals are distinct and help to reduce the potential for misattribution of adverse events. Because two products would share a common 'core' portion of the non-proprietary name, this policy would also allow for the aggregation of data to assess class-wide safety issues. 


\section{Conclusion}

WHO should thus develop policies requiring distinct non-proprietary names for biosimilars to best serve the interest of public health. Swift action is critical. Although biosimilars have a rapidly expanding global footprint, the number of approved biosimilars is still limited enough that a new approach for naming biologicals, even if applied only prospectively, will result in significantly improved consistency. WHO should also investigate the use of distinct non-proprietary names for all biologicals. These policies would not require drastic changes to existing naming processes or prescribing and dispensing systems, but would reduce the potential for misattribution of adverse events and would improve product identification and transparency. These goals are becoming more critical as the number of approved biologicals increases. In the coming years, many new biologicals will enter the market, and some will be biosimilar, some may be interchangeable, and some will not undergo any comparability testing with another biological. A consistent, clear naming policy is critical to preventing confusion.

In addition to $\mathrm{WHO}$, national regulatory authorities will play key roles as well. First, because WHO does not itself have the authority to make the INN process mandatory, national regulatory authorities should help facilitate global consistency by adopting policies that encourage all biological applicants to apply for an INN from WHO. Second, coordination between $\mathrm{WHO}$ and national regulatory authorities is also critical. Applicants are more likely to apply for an INN if the process is efficient and does not interfere with applicant review and approval timelines by national regulatory authorities. Finally, even if WHO adopts a unique identifier approach, this policy will have little effect if the identifier is not used on prescriptions, patient records, product labelling and advertising, adverse event reports, and other records. National regulatory authorities can therefore play a key role in educating healthcare professionals, manufacturers, and other stakeholders on the importance of including the unique identifier in all contexts.

Other tools should be used to complement these efforts and may help ensure the clear identification of biologicals and effective pharmacovigilance. For example, the use of distinct brand names may also help to distinguish between products. All biosimilar products in the European Union have had a distinct product name, and many stakeholders have suggested that the use of distinct brand names for biosimilars will eliminate the need for distinct non-proprietary names. However, not all jurisdictions have the authority to require that products bear a brand name. For example, in the US, FDA does not have explicit statutory authority to require that all biosimilars use a distinct brand name. There are also some jurisdictions that prohibit or discourage prescribing certain products by brand name, and many others - including the US - that do not mandate prescribing by brand name, so the use of distinct non-proprietary names becomes critical.

In addition, it is critically important to educate patients, physicians, and other stakeholders on the importance of reporting more product-identifying information in adverse event reports and prescribing and dispensing records, such as brand name, non-proprietary name, manufacturer name, and lot number.

Biologicals are revolutionizing healthcare, and biosimilars may offer additional options for patients. But in order for these products to offer the greatest benefit to patients, we must be able to accurately identify them and efficiently track any applicable adverse events. In this way, the simple concept of a name is critical to protecting patients around the world.

Competing interests: Ms Alexander is an employee of AbbVie, Inc. She has no conflict of interest that is directly relevant to the content of this manuscript.

Provenance and peer review: Not commissioned; internally peer reviewed.

\section{References}

1. World Health Organization. 56th Consultation on International Nonproprietary Names (INN) for Pharmaceutical Substances. Geneva, 15-17 April 2013. Executive Summary. September 2013 [homepage on the Internet]. 2013 Oct [cited 2014 Jan 9]. Available from: http://www.who.int/medicines/services/ inn/56th_Executive_Summary.pdf
2. European Medicines Agency. Silapo: EPAR-Scientific Discussion.January 2008[homepageon theInternet]. 2008 Jan [cited 2014 Jan 9]. Available from: http:// www.ema.europa.eu/docs/en_GB/document_ library/EPAR_-_Scientific_Discussion/human/ 000760/WC500050914.pdf

3. European Medicines Agency. Assessment Report: Remsima. 27 June 2013 [homepage on the Internet]. 2014 [cited 2014 Jan 9]. Available from: http:// www.ema.europa.eu/docs/en_GB/document_ library/EPAR___Public_assessment_report/human/ 002576/WC500151486.pdf

4. Australian Government. Department of Health. Therapeutic Goods Administration. Evaluation of biosimilars. 30 July 2013 [homepage on the Internet]. 2014 [cited 2014 Jan 9]. Available from: http://www.tga.gov.au/ industry/pm-argpm-biosimilars-00.htm\#.Usn2XjOA3Dd

5. Arato T. Recent regulations of biosimilars in Japan. Pharmaceuticals and Medical Devices Agency. 47th AnnualMeeting,DIA2011;2011Jun19-23,Chicago,USA. Available from: http://www.pmda.go.jp/regulatory/ file/english_presentation/biologics/B-E1arato.pdf

6. Derbyshire M. Biosimilar development and regulation in Japan. Generics and Biosimilars Initiative Journal (GaBI Journal). 2013;2(4):207-8. doi:10.5639/ gabij.2013.0204.055

7. U.S. Food and Drug Administration. Public Health Service. Department of Health and Human Services. FDA Biological Product Naming Working Group, FDA Memorandum, BLA 125294 [xxx]-filgrastim. 2 August 2012 [homepage on the Internet]. [cited 2014 Jan 9]. Available from: http://www.accessdata.fda.gov/drugsatfda_docs/ nda/2012/125294Orig1s000NameR.pdf

8. Industry Standard Research. Alliance for Safe Biologic Medicine-Prescriber survey [homepage on the Internet]. 2012 Aug 31 [cited 2014 Jan 9]. Available from: http://safebiologics.org/resources/wp-content/ uploads/2012/09/ASBM-Survey-1.pdf

9. Alliance for Safe Biologic Medicines. ASBM presents new European survey findings on biosimilars and the importance of nonproprietary naming [homepage on the Internet]. 2013 Nov 22 [cited 2014 Jan 9]. Available from: http://safebiologics.org/ resources/2013/11/asbm-presents-new-europeansurvey-findings-on-biosimilars-and-the-importanceof-nonproprietary-naming

10. Prescriber Survey. ZS Associates on behalf of AbbVie. 2013. Data on file at AbbVie.

11. Biosimilar medicines and safety: new challenges for pharmacovigilance. WHO Drug Information. 2009;23(2):87-91. Available from: http://apps.who. int/medicinedocs/documents/s16240e/s16240e.pdf

12. Lietzan $\mathrm{E}$, et al. Biosimilar naming: how do adverse event reporting data support the need for distinct nonproprietary names for biosimilars? FDLI's Food and Drug Policy Forum. 2013;3(6):1-20. DOI: 10.5639/gabij.2014.0301.005

Copyright $\odot 2014$ Pro Pharma Communications International 